\title{
Theoretische Untersuchungen zur Strömung im glatten Rohr
}

\author{
Wolfgang Frie \\ Forschungslaboratorium Erlangen der Siemens Aktiengesellschaft \\ (Z. Naturforsch. 23 a, 1478-1487 [1968]; eingegangen am 12. Juli 1968)
}

\begin{abstract}
Unter Benutzung des vollständigen Ansatzes für den Zusammenhang zwischen der Schubspannung und dem Geschwindigkeitsgradienten wird durch eine plausible Festlegung der radialen Verteilung des Mischungsweges und durch Einführung eines ,Turbulenzgrades" als Anregungsgesetz für die turbulente Energie eine analytische Lösung hergeleitet, die das Strömungsverhalten im glatten Rohr für alle Reynolds-Zahlen beschreibt. Diese Lösung enthält das Hagen-Poiseuillesche Gesetz der laminaren Strömung und das Prandtl-v.Kármánsche Gesetz der turbulenten Strömung als Grenzfälle, gibt aber auch das Utbergangsgebiet recht gut wieder.
\end{abstract}

\section{Problemstellung}

Will man einen möglichst vollständigen Überblick über die Arbeit gewinnen, die bisher auf dem Gebiet der Strömung in Rohren geleistet wurde, dann empfiehlt es sich, sowohl die die technischen Anwendungen betreffenden Lehrbücher ${ }^{1,2}$ als auch die der theoretischen Physik ${ }^{3}$ zu studieren. Danach ergibt sich das folgende Bild.

Die auf REYNoLDS ${ }^{4}$ zurückgehende Erkenntnis, daß man nicht nur das Hagen-Poiseu illesche Gesetz $^{5,6}$ der laminaren Strömung, sondern die gesamte Strömungskennlinie als Beziehung zwischen der Widerstandszahl $\lambda$ und der Reynolds-Zahl Re in normierter Form darstellen kann, ist durch eine Reihe von Messungen bestätigt worden ${ }^{7,8}$.

Schließlich gelang es PrandtL ${ }^{9,10}$ und v. KáRMÁN ${ }^{11}$, eine verhältnismäßig einfache analytische Formel herzuleiten, die die Strömungskennlinie im turbulenten Teil erstaunlich gut wiedergibt. Der Gedanke, daß diese gute Übereinstimmung Zufall sein könnte, liegt natürlich nicht gleich auf der Hand. Der Verfasser neigt jedoch zu der Ansicht,

1 B. Eck, Technische Strömungslehre, Springer-Verlag, 6. Auflage, Berlin 1961.

2 C. Pfleiderer, Die Kreiselpumpe für Flüssigkeiten und Gase, Springer-Verlag, 5. Auflage, Berlin 1961.

3 A. Sommerfeld, Vorlesungen über theoretische Physik, Bd. II, Akademische Verlagsgesellschaft Geest und Portig, 5. Auflage, Leipzig 1964, § 38 .

4 O. Reynolds, Phil. Trans. Roy. Soc. London 174, 935 [1883]; 186, 123 [1895].

5 G. Hagen, Poggendorffs Ann. 46, 423 [1839].

6 J. L. Poiseuille, C. R. Acad. Sci. Paris 11 [1840]; 12 [1841].

7 T. E. Stanton u. Pannel, Phil. Trans. (A) 214, 199 [1914].

8 J. Nikuradse, Gesetzmäßigkeit der turbulenten Strömungen in glatten Rohren, VDI-Forschungsheft $\mathbf{3 5 6}$ [1932]. daß dies hier in der Tat so ist. Denn beim Übergang von der in der vorliegenden Arbeit zu beschreibenden Lösung zur Prandtl-v. Kármánschen Formel als ihrer Asymptote begeht man zwei Fehler, die sich oberhalb der kritischen Reynolds-Zahl weitgehend kompensieren.

Die Verhältnisse sind im Übergangsgebiet sicherlich verwickelter als sie die einfachen Ansätze von Prandtl und v. Kármán vermuten lassen. Es handelt sich hier offensichtlich um eine Anregung energetisch höherer turbulenter Zustände, was eine Energiestatistik für die einzelnen Elementarbereiche der Strömung erforderlich macht. Aufbauend auf den Arbeiten von TAYLOR ${ }^{12}$, Simmons und SALTER $^{13}$, v. WeIZsäckeR ${ }^{14}$ und HeIsenberg 15 wird an einer derartigen Statistik der ,Turbulenzelemente" noch gearbeitet ${ }^{16}$. Ein Gesetz ähnlich dem für den Dissoziationsgrad bei der thermischen Anregung von Molekülen, das die statistische Verteilung der laminaren und turbulenten Elementarbereiche angibt, ist jedoch nicht bekannt.

Da diese Untersuchungen recht kompliziert sind, soll in der vorliegenden Arbeit mit Hilfe einiger

9 L. Prandth, Verhandlungen des 2. internationalen Kongresses für techn. Mechanik [1927].

10 L. Prandtl, Z. VDI 77, 105 [1933].

11 Th. v. Kármán, Nachr. Ges. Wiss. Göttingen 1930, 58.

12 G. J. TAYlor, Proc. Roy. Soc. London A 151, 421 [1935]; 164, 476 [1938].

13 L. F. Simmons u. C. Salter, Proc. Roy. Soc. London A 165, 73 [1938].

14 C. F. v. Weizsäcker, Z. Phys. 124, 614 [1948].

15 W. Heisenberg, Z. Phys. 124, 628 [1948]; Proc. Roy. Soc. London A 195, 402 [1948/49].

16 J. Neumann, Phys. Fluids 10, Heft 9/II [1967]; Proc. Intern. Symposium on Boundary Layers and Turbulence S 66 . 
plausibler phänomenologischer Ansätze eine vollständige Beschreibung der Strömungskennlinie in glatten Rohren gegeben werden.

\section{Die Grundgleichungen}

$L$ sei die Länge des durchströmten Rohres, $R$ sein Radius, $r$ die radiale Ortskoordinate. $\Delta p$ ist die angelegte Druckdifferenz und $\tau$ die Schubspannung. Das Kräftegleichgewicht ist bei stationärer Strömung gegeben durch die Gleichung

oder integriert

$$
\frac{\mathrm{l}}{r} \frac{\mathrm{d}}{\mathrm{d} r} r \tau=\frac{\Delta p}{L}
$$

$$
\tau=\frac{\Delta p}{L} \frac{r}{2} .
$$

Der Zusammenhang zwischen der Schubspannung $\tau$ und dem Geschwindigkeitsgradienten in radialer Richtung $\mathrm{d} v / \mathrm{d} r$ wird in der von PrandtL ${ }^{9}$ angegebenen Form angesetzt:

$$
\tau=-\eta \frac{\mathrm{d} v}{\mathrm{~d} r}+\varrho l^{2}\left(\frac{\mathrm{d} v}{\mathrm{~d} r}\right)^{2} .
$$

$\varrho$ ist die Dichte, $\eta$ die Zähigkeit des strömenden Mediums. Der zweite Term der rechten Seite von (2) beschreibt den turbulenten Zusatz zur Schubspannung. Dabei ist $l$ der sogenannte ,Mischungsweg". Er beschreibt anschaulich etwa die Ausdehnung der in Abschnitt I erwähnten Turbulenzelemente. Mit $\bar{v}$ als der mittleren Geschwindigkeit seien nun die folgenden normierten Größen eingeführt:

$$
\begin{aligned}
R e & =\frac{\varrho \bar{v} 2 R}{\eta} \equiv \text { Reynolds-Zahl }, \\
\lambda & =\frac{\Delta p}{\frac{1}{2} \varrho \bar{v}^{2}} \frac{2 R}{L} \equiv \text { Widerstandszahl }, \\
u & =r / R \equiv \text { relativer Radius, } \\
w & =v / \bar{v} \equiv \text { relative Geschwindigkeit, } \\
s & =l / R \equiv \text { relativer Mischungsweg. }
\end{aligned}
$$

Damit bekommt Gl. (2) die Form:

$$
\frac{1}{8} \lambda \operatorname{Re} u=-2 \frac{\mathrm{d} w}{\mathrm{~d} u}+\operatorname{Re}^{2}\left(\frac{\mathrm{d} w}{\mathrm{~d} u}\right)^{2} .
$$

Außerdem ist: $\quad w(u)=-\int_{u}^{1} \mathrm{~d} u^{\prime} \frac{\mathrm{d} w\left(u^{\prime}\right)}{\mathrm{d} u^{\prime}}$.

Da $\bar{v}=\left(2 / R^{2}\right) \int_{0}^{R} \mathrm{~d} r r v(r)$ ist, beschreibt die Gleichung

$$
2 \int_{0}^{1} \mathrm{~d} u u w(u)=-\int_{0}^{1} \mathrm{~d} u u^{2} \frac{\mathrm{d} w}{\mathrm{~d} u}=1
$$

die Strömungskennlinie, also den Zusammenhang zwischen $\lambda$ und $R e$.

Für das Verhältnis von Maximalgeschwindigkeit $v_{0}$ zur mittleren Geschwindigkeit $\bar{v}$ bekommt man schließlich noch:

$$
v_{0} / \bar{v}-1=-\int_{0}^{1} \mathrm{~d} u\left(1-u^{2}\right) \frac{\mathrm{d} w}{\mathrm{~d} u} .
$$

\section{Das Hagen-Poiseuille'sche Gesetz der laminaren Strömung}

Für $s=0$ folgt für die laminare Strömung

$$
\text { aus }(6): \quad \lambda \cdot R e=64
$$

und aus $(5): \quad w(u)=2\left(1-u^{2}\right) ; v_{0} / \bar{v}=2$.

\section{Das Prandtl-v. Kármánsche Gesetz der turbulenten Strömung ${ }^{10,} 11$}

Da an der Rohrwand eine laminare Grenzschicht existiert, muß der Mischungsweg an der Wand gleich Null werden und von dort zur Rohrmitte hin zunächst etwa linear ansteigen. Außerhalb der laminaren Grenzschicht ist das erste Glied auf der rechten Seite von Gl. (4) bei großen ReynoldsZahlen vernachlässigbar. Da nur die wandnahen Bereiche einen wesentlichen Beitrag zum Integral (6) liefern, kann man außerdem die Schubspannung konstant halten. So bekommt man mit

$$
l=\varkappa y, \quad y=R-r \equiv \text { Wandabstand }
$$

und $u \approx 1$

aus (4):

$$
\begin{aligned}
& \frac{1}{8} \operatorname{Re} \lambda=\operatorname{Re}^{2} y^{2}\left(\frac{\mathrm{d} w}{\mathrm{~d} y}\right)^{2} \text { oder } \frac{\mathrm{d} w}{\mathrm{~d} y}=(1 / \varkappa) \sqrt{\lambda / 8} \cdot 1 / y, \\
& \text { also } \quad w(y)=(1 / \varkappa) \sqrt{\lambda / 8} \ln \left(y / y_{0}\right) .
\end{aligned}
$$

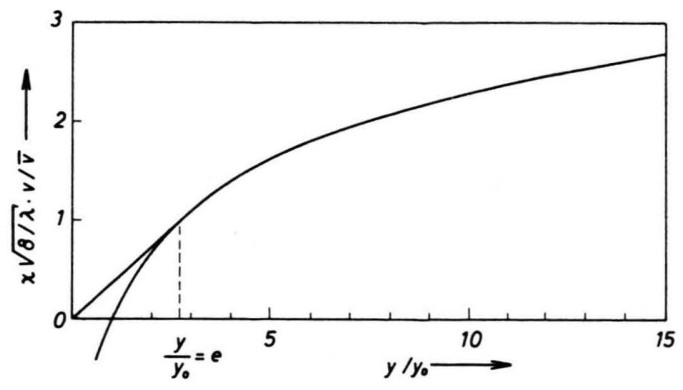

Abb. 1. Logarithmische Geschwindigkeitsverteilung bei voller Turbulenz. 
Diese Lösung für die Geschwindigkeitsverteilung ist in Abb. 1 dargestellt. Da sie für $y \rightarrow 0$ gegen $-\infty$ geht, muß sie für $y<y^{*}=e y_{0}$ durch die eingezeichnete Nullpunktstangente ersetzt werden. Aus Gl. (7) ergibt sich zunächst für die Maximalgeschwindigkeit $v_{0}$ :

$$
\begin{aligned}
v_{0} / \bar{v}-1 & =w(y=R)-1 \\
= & \frac{1}{x} \sqrt{\frac{\lambda}{8}} \int_{0}^{R} \frac{\mathrm{d} y}{R} \frac{y}{R}\left(2-\frac{y}{R}\right) \frac{R}{y}, \\
0 \quad v_{0} / \bar{v}-1 & =(1 / x) \sqrt{\lambda / 8} \cdot 3 / 2 .
\end{aligned}
$$

also

Da aus (11) folgt:

$$
w(y=R)=(1 / x) \sqrt{\lambda / 8} \ln \left(R / y_{0}\right),
$$

ergibt der Vergleich mit (12):

$$
1 / \sqrt{\lambda}=(1 / \sqrt{8} x) \ln \left(R e^{-3 / 2} / y_{0}\right) .
$$

Dasselbe Ergebnis bekommt man aus Gl. (6) mit Hilfe der Abb. 1 für den Grenzübergang $y_{0} \rightarrow 0$. Gl. (13) beschreibt also die Strömungskennlinie, wenn die Größe $y_{0}$ festgelegt ist. Dazu kann man folgendermaßen argumentieren:

Die wandnahen Bereiche wissen nichts von der Größe des Rohrradius $R$. Die einzige Länge, die bei der Festlegung von $y_{0}$ eine Rolle spielt, muß sich aus den Größen $\tau, \varrho$ und $\eta$ zusammensetzen. Setzt man für diese charakteristische Länge $l_{\mathbf{c}}$ an:

$$
l_{\mathrm{c}}=(1 / \sqrt{32}) \eta^{\mathrm{a}} \tau_{\mathrm{w}}^{\mathrm{b}} \varrho_{\mathrm{c}} \quad \text { mit } \quad \tau_{\mathrm{w}}=\tau(y=0),(14 \mathrm{a})
$$

dann bekommt man durch Dimensionsvergleich:

$$
l_{\mathrm{c}}=(1 / \sqrt{32})\left(\eta / \sqrt{\tau_{\mathrm{w}} \varrho}\right)=R /(\operatorname{Re} \sqrt{\bar{\lambda})} .
$$

So hat man für $y_{0}$ anzusetzen:

$$
y_{0} e^{3 / 2}=c_{0} l_{\mathrm{c}}=c_{0} \cdot R /(\operatorname{Re} \sqrt{\lambda})
$$

und bekommt für die Strömungskennlinie die Formel

$$
1 / \sqrt{\lambda}=(1 / \varkappa / \overline{8}) \ln \left(\operatorname{Re} \sqrt{\lambda /} c_{0}\right) .
$$

$c_{0}$ ist dabei wie $\varkappa$ eine Konstante. Mit

$$
\varkappa=0,4 \text { und } c_{0}=2,51
$$

kann man die Formel (16) optimal an die Messungen anpassen. Die Prandtl-v. Kármánsche Formel gibt die Strömungskennlinie im turbulenten Bereich ausgezeichnet wieder (siehe Abb. 3), und auch die logarithmische Geschwindigkeitsverteilung ist experimentell bis in Achsennähe bestätigt ${ }^{10}$. Obwohl die gute Übereinstimmung wohl mehr Zufall ist, liegt die Bedeutung der Formeln (12) und (16) für die vorliegenden Untersuchungen darin, daß sie zwei Grenzgesetze angeben, in die die herzuleitenden genaueren Formeln für große Reynolds-Zahlen asymptotisch übergehen müssen.

\section{Die allgemeine Lösung}

\section{a) Voraussetzungen}

Die Hauptschwierigkeit bei der Behandlung der Gl. (2) bzw. (4) liegt in der Festlegung des Mischungsweges $l$. v. KárMáN ${ }^{11}$ hat zur Beschreibung der Turbulenz eine Störgleichung hergeleitet und durch Diskussion dieser Gleichung als Erweiterung von (10) den folgenden Ausdruck für den Mischungsweg angegeben:

$$
l=\varkappa \cdot \frac{\mathrm{d} v}{\mathrm{~d} r} / \frac{\mathrm{d}^{2} v}{\mathrm{~d} r^{2}},
$$

der auch in der abgewandelten Form

$$
l=\varkappa\left|\frac{\mathrm{d} v}{\mathrm{~d} r} / r \frac{\mathrm{d}}{\mathrm{d} r} \frac{1}{r} \frac{\mathrm{d} v}{\mathrm{~d} r}\right|
$$

noch heute benutzt wird ${ }^{17,18}$. Setzt man voraus, daß sich die Geschwindigkeitsverteilung in der Rohrmitte parabolisch verhält

$$
v=v_{0}-v_{2} r^{2}+v_{3} r^{3}+\cdots,
$$

dann liefert der Ansatz (18a) für den Mischungsweg:

Der Ausdruck

$$
l=x r+\cdots
$$

$$
v_{r}=l(\mathrm{~d} v / \mathrm{d} r)
$$

hat die Bedeutung der mittleren radialen Störgeschwindigkeit. Für diese ergibt sich dann:

$$
v_{r} \sim r^{2},
$$

was im Widerspruch steht zu der plausiblen Annahme, daß $v_{r}$ in der Rohrachse durch eine ungerade Potenz von $r$ dargestellt wird. v. KÁRMÁN 11 bekommt bei der Diskussion des Ansatzes (18a) dann auch eine Singularität in der Rohrmitte. Diesen Schönheitsfehler des Widerspruchs in der plausiblen Symmetrievorstellung vermeidet der Ansatz (18b). Dennoch scheinen diese Ansätze nicht viel weiter zu führen als die einfache Prandtlsche Form

17 J. K. Vennard, One Dimensional Flow, Handbook of Fluid Dynamies, Sect. 3, S. 3-15, McGraw-Hill Book Co. Ine. New York 1961 .

18 R. SCHRÖDER, VDI-Z. 110, 93 [1968]. 
$l=x y$ aus Gl. (10), weshalb TIEDT ${ }^{19}$ in seinen Untersuchungen auch auf diese zurückgreift. Außerdem erscheint ein Ansatz dieser Art noch aus einem anderen Grunde dem Verfasser bedenklich. Die Thermodynamik irreversibler Prozesse 20, 21 liefert bei der

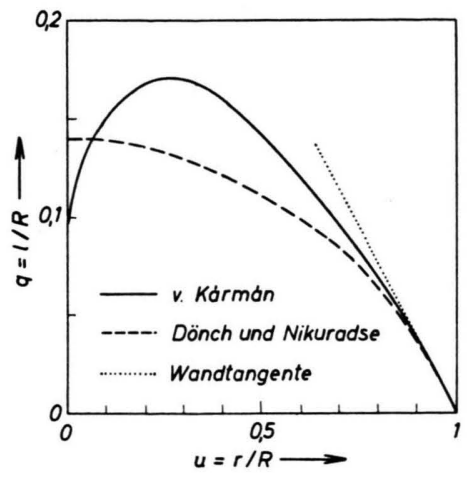

Abb. 2. Radiale Verteilung des Mischungsweges nach v. KáRMáN ${ }^{11}$ und DöNCH ${ }^{22} /$ NIKURADSE $^{23}$.

Herleitung der phänomenologischen Gleichungen die Aussage, daß der Spannungstensor eine Funktion des Geschwindigkeitsgradienten ist. In diesem Sinne ist Gl. (2) eine Entwicklung dieser Funktion bis zum quadratischen Glied, wobei der vor dem Quadrat des Geschwindigkeitsgradienten stehende Koeffizient logischerweise von Geschwindigkeitsgradienten unabhängig sein sollte. Hier soll deshalb auf einen anderen Ansatz zurückgegriffen werden, der sich bereits bei PRANDTL ${ }^{10}$ findet, nämlich daß der relative Mischungsweg $s=l / R$ eine Funktion des relativen Radius $u=r / R$ und der ReynoldsZahl $R e$ ist, wobei für große Reynolds-Zahlen der relative Mischungsweg nur vom relativen Radius $u$ allein abhängt:

$$
s=q(u) .
$$

Dabei ist zu fordern, daß $s$ am Rande gemäß (10) auf Null abfällt. Man kann dann $\mathrm{d} w / \mathrm{d} u$ aus (4) berechnen

$$
\frac{\mathrm{d} w}{\mathrm{~d} u}=-\frac{1}{8} \operatorname{Re} \lambda \frac{u}{1+\sqrt{1+\operatorname{Re}^{2} \frac{1}{8} \lambda s^{2} u}}
$$

und bekommt für die Strömungskennlinie aus (6):

$$
\frac{1}{\operatorname{Re} \lambda}=\frac{1}{8} \int_{0}^{1} \mathrm{~d} u \frac{u^{3}}{1+\sqrt{1+\operatorname{Re}^{2} \frac{1}{8} \lambda s^{2} u}}
$$

19 W. TindT, Chemiker Ztg./Chem. Apparatur 90, 813 [1966]; 91, 17, 149, 299 [1967].

20 S. R. DE Groot, Thermodynamics of Irreversible Processes, North Holland Publishing Comp. Amsterdam $1951,1959$.

21 J. Meixner u. H. G. Reik, Handbuch der Physik, Bd. III/2, Springer-Verlag, Berlin 1959. und für die Maximalgeschwindigkeit aus (7):

$$
\frac{v_{0}}{\bar{v}}-1=\frac{1}{8} \operatorname{Re} \lambda \int_{0}^{1} \mathrm{~d} u \frac{u\left(1-u^{2}\right)}{1+\sqrt{1+R^{2} \frac{1}{8}} \lambda s^{2} u} .
$$

Im folgenden wird zunächst untersucht, welche radiale Verteilung des Mischungsweges bei großen Reynolds-Zahlen Gl. (21) in die Prandtl-v. Kármánsche Formel (13) und (22) in (12) überführt. Dann wird eine analytische Lösung hergeleitet. Zum Schluß wird die Abhängigkeit des Mischungsweges von der Reynolds-Zahl berücksichtigt. Dies geschieht am einfachsten durch einen Produktansatz für den Mischungsweg:

$$
s=q(u) \cdot g(R e) .
$$

$g(R e)$ ist dabei ein Faktor, der im Übergangsgebiet von Null auf Eins ansteigt. Er beschreibt also gewissermaßen den Turbulenzanteil der Strömung und soll als ,Tubulenzgrad“ bezeichnet werden.

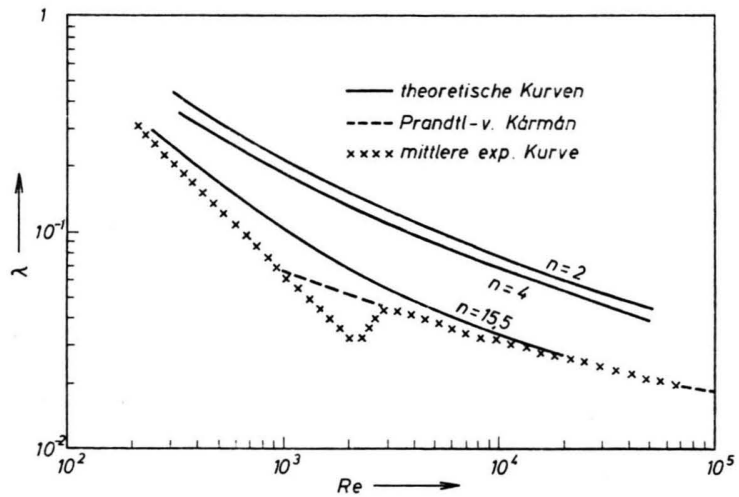

Abb. 3. Theoretische Strömungskennlinien für $l / R=(\varkappa / n)$ - $\left(1-u^{n}\right)$ mit der Formel von PrandtL und v. Kármán und einer Mittelkurve durch die Messungen von Stanton und PanNeL ?

\section{b) Bestimmung der radialen Verteilung des Mischungsweges für volle Turbulenz}

In Abb. 2 ist neben der radialen Verteilung des Mischungsweges nach v. KÁRMÁN ${ }^{11}$ noch diejenige eingetragen, die von DöNch ${ }^{22}$ und NIKURADSE ${ }^{23}$ ermittelt wurde. Diese sieht recht plausibel aus und regt dazu an, zunächst einmal den Ansatz

$$
s=(\varkappa / n)\left(1-u^{n}\right)
$$

22 F. DöNch, Divergente und konvergente turbulente Strömungen mit kleinen Öffnungswinkeln, Diss. Göttingen 1925, VDI-Forschungsheft 282 [1926].

23 J. NikURADSE, Strömung des Wassers in konvergenten und divergenten Kanälen, VDI-Forschungsheft $\mathbf{2 8 9}$ [1929]. 


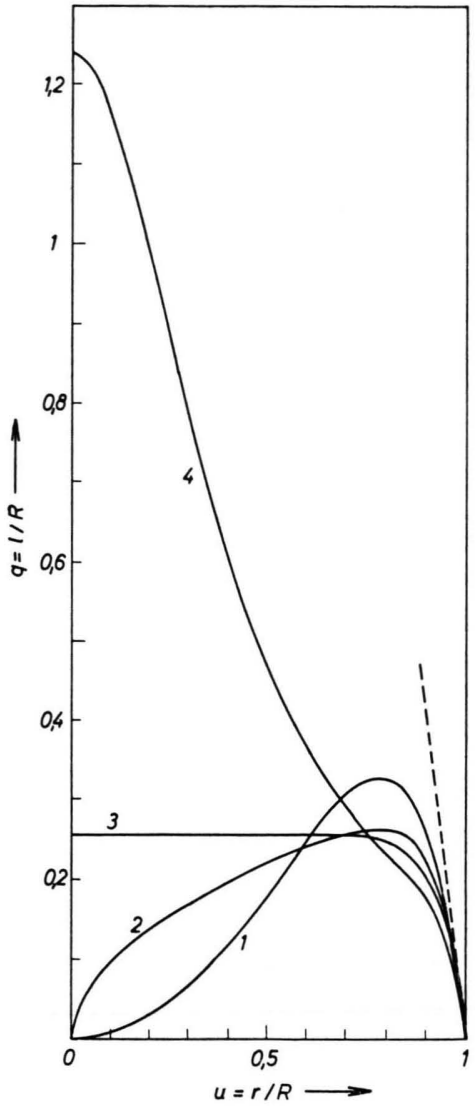

Abb.4. Eine Auswahl von Radialverteilungen des Mischungsweges, die die gemessene Strömungskennlinie bei großen Reynolds-Zahlen richtig wiedergeben.

$\mathrm{zu}$ versuchen und die Gl. (21) für verschiedene $n$ numerisch mit Hilfe einer elektronischen Datenverarbeitungsanlage zu integrieren. Das Ergebnis für $n=2, n=4$ und $n=15,5$ zeigt Abb. 3. Dort sind zum Vergleich die Prandtl-v. Kármánsche Formel und eine Mittelkurve durch die Messungen von Stanton und Pannel ${ }^{7}$, wie sie bei Eck ${ }^{1}$, Abb. 119, aufgezeichnet sind, mit angegeben. Man sieht: erst für $n=15,5$ liegt die Kurve bei großen ReynoldsZahlen richtig.

Natürlich gibt es noch andere Verteilungen, die die Messungen bei großen Reynolds-Zahlen richtig wiedergeben. Eine repräsentative Auswahl derartiger Radialverteilungen des Mischungsweges zeigt Abb. 4. Alle liefern etwa dieselbe Strömungskennlinie (Abb. 5). Sie unterscheiden sich jedoch wesentlich im Verhältnis von Maximalgeschwindigkeit $v_{0}$ zur mittleren Geschwindigkeit $\bar{v}$. In Abb. 6 sind diese Werte zusammen mit der Prandtl-v. Kármánschen Lösung nach Gl. (12) und dem laminaren

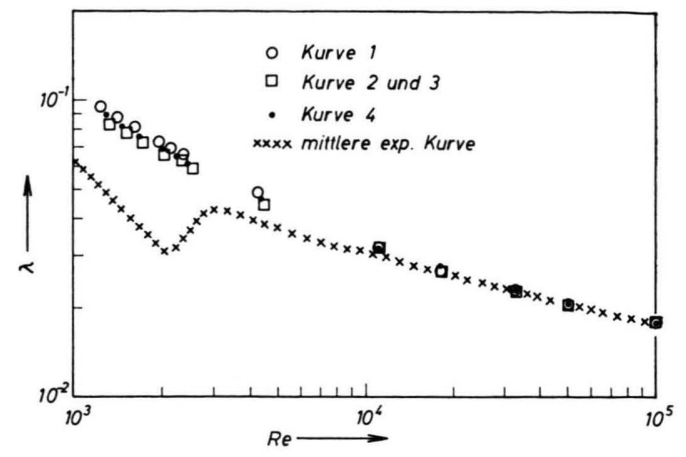

Abb. 5. Die theoretischen Strömungskennlinien zu den Verteilungen von Abb. 4.

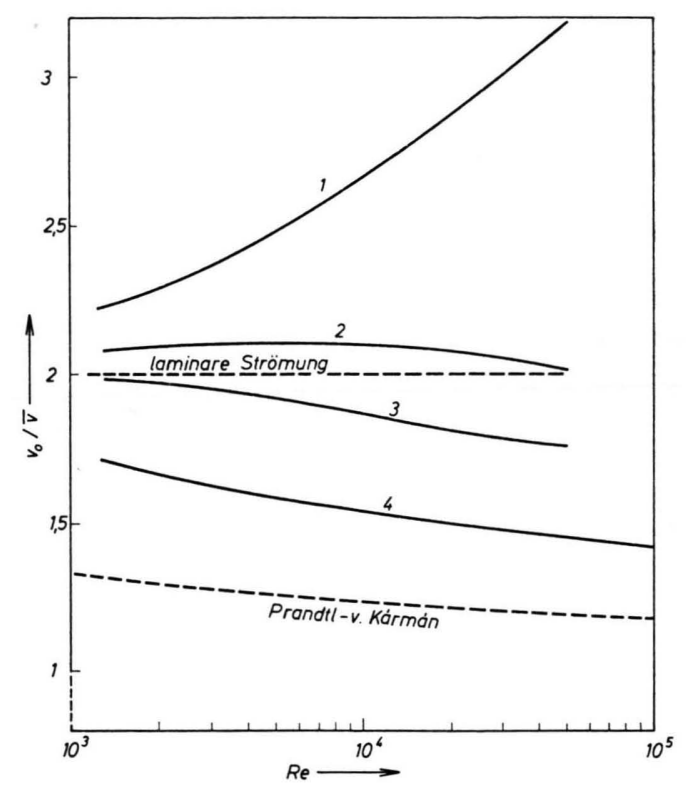

Abb. 6. Das Verhältnis von Maximalgeschwindigkeit $v_{0}$ zur mittleren Geschwindigkeit $\bar{v}$ für die Verteilungen von Abb. 4.

Wert $v_{0} / \bar{v}=2$ aufgetragen. Man sieht, daß von allen diesen Verteilungen des Mischungsweges nur die Kurve Nr. 4 in die richtige Größenordnung kommt. Man braucht also eine Verteilung, die an der Wand sehr bald von der Randtangente $l=x y$ abbiegt und dann nach einem flachen Zwischenstück noch einmal stark ansteigt. Abb. 7 zeigt einige Verteilungen, die die geforderten Bedingungen erfüllen (Abb. 8 und 9).

Diese Ergebnisse legen es nahe, zur Herleitung einer analytischen Lösung den folgenden Ansatz für den Mischungsweg zu machen: 


$$
q(u)=\left\{\begin{array}{l}
q_{1}\left(u / u_{1}\right)^{m / 2} \\
\text { für } 0<u<u_{1} \\
\text { mit } m=-1,0,+1 \\
q_{2}\left(u_{2} / u\right)^{1 / 2} \\
\text { für } u_{1}<u<u_{2} \\
x(1-u) \approx(\varkappa / 2 n)\left(1-u^{2 n}\right) \\
\text { für } u_{2}<u<1 \text { mit } u_{2} \approx 1 \\
\text { und } x=0,4 .
\end{array}\right.
$$

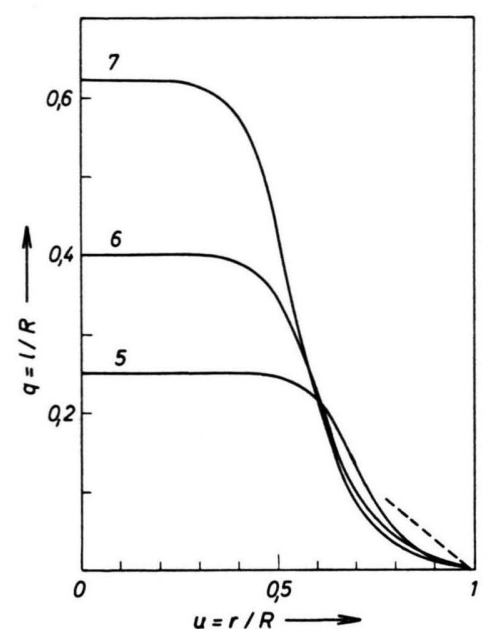

Abb. 7. Einige weitere Radialverteilungen des Mischungsweges.

Dabei ist

$$
\begin{aligned}
& q_{2}=\varkappa\left(1-u_{2}\right) \approx(\varkappa / 2 n)\left(1-u_{2}^{2 n}\right) \quad \text { und } \\
& q_{1}>q_{2} \sqrt{u_{2} / u_{1}} .
\end{aligned}
$$

In dieser Arbeit soll nur der Fall $m=0$, der einen konstanten Wert für den Mischungsweg im achsennahen Bereich beschreibt, untersucht werden. Die Fälle $m=-1$ und $m=+1$ führen zwar zu etwas

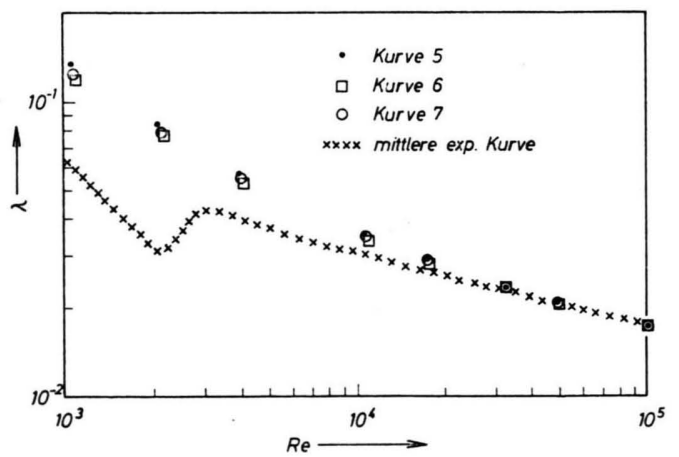

Abb. 8. Die theoretischen Strömungskennlinien zu den Verteilungen von Abb. 7.

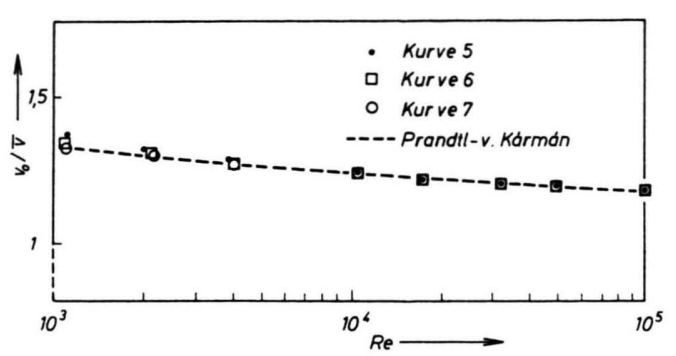

Abb. 9. Das Verhältnis von Maximalgeschwindigkeit $v_{0}$ zur mittleren Geschwindigkeit $\bar{v}$ für die Verteilungen von Abb. 7.

einfacheren analytischen Ausdrücken, sind aber nicht so plausibel wie der Fall $m=0$, der allein ein reguläres Verhalten in der Achse kennzeichnet. Außerdem ist der Vorteil nicht so groß, daß er den dreifachen Rechenaufwand, der zur Diskussion aller drei Fälle nötig wäre, rechtfertigt.

Die Integration der Gln. (21) und (22) mit Hilfe des Ansatzes (24a, b) ist elementar. Die wichtigsten Schritte seien kurz skizziert:

Man braucht die Integrale

$$
\begin{aligned}
& J_{n}=\frac{1}{8} \int \mathrm{d} u \frac{u^{2 n-1}}{1+V^{1+R e^{2} \frac{1}{8} \lambda g^{2} q^{2} u}} \\
& \text { für } n=1 \text { und } n=2 .
\end{aligned}
$$

Im Bereich $u_{2}<u<1$ bekommt man mit Hilfe der Substitution

$$
\begin{aligned}
z= & \operatorname{Re} \sqrt{\lambda / 8} g q R e \sqrt{\lambda / 8} g \varkappa / 2 n\left(1-u^{2 n}\right) ; \\
& \mathrm{d} z \approx-\operatorname{Re} \sqrt{\lambda / 8} g \varkappa u^{2 n-1} \mathrm{~d} u ; \quad \text { (25a) } \\
& z_{2}=\operatorname{Re} \sqrt{\lambda / 8} g q_{2}
\end{aligned}
$$

und $u \approx 1$ :

$$
\begin{aligned}
J_{n} & =\frac{1}{R e \sqrt{\lambda} g \sqrt{8} \varkappa} \int_{0}^{z_{2}} \frac{\mathrm{d} z}{1+\sqrt{1+z^{2}}} \\
& =\frac{1}{\operatorname{Re} \sqrt{\lambda} g \sqrt{8} \varkappa}\left\{\ln \left(z_{2}+\sqrt{\left.1+z_{2}^{2}\right)}-\frac{z_{2}}{1+\sqrt{1+z_{2}^{2}}}\right\} .\right.
\end{aligned}
$$

Im Bereich $u_{1}<u<u_{2}$ hat man einfach:

$$
J_{n} \approx \frac{1}{8} \int_{u_{1}}^{u_{2}} \mathrm{~d} u \frac{u^{2 n-1}}{1+V^{1+z_{2}^{2}}}=\frac{1}{16 n} \frac{u_{2}^{2 n}-u_{1}^{2 n}}{1+\sqrt{1}+z_{2}^{2}} .
$$

Für $0<u<u_{1}$ wird die Integration etwas komplizierter. Hier führt $q=q_{1}=$ const. und die Substitution

$$
\begin{aligned}
& R_{e}^{2}(\lambda / 8) q_{1}^{2} g^{2} u=w(w+2) \\
& R_{e}^{2}(\lambda / 8) q_{1}^{2} g^{2} u_{1}=w_{1}\left(w_{1}+2\right)
\end{aligned}
$$


auf das Integral

$J_{n}=\frac{1}{4} \frac{u_{1}^{2 n}}{w_{1}^{2 n}\left(w_{1}+2\right)^{2 n}} \int_{0}^{w_{1}} \mathrm{~d} w(w+1) w^{2 n-1}(w+2)^{2 n-2}$,

also :

$$
\begin{aligned}
& J_{1}=\frac{1}{8} \frac{u_{1}^{2}}{\left(w_{1}+2\right)^{2}}\left(1+\frac{2}{3} w_{1}\right) \\
& J_{2}=\frac{1}{4} \frac{u_{1}^{4}}{\left(w_{1}+2\right)^{4}}\left(1+\frac{8}{5} w_{1}+\frac{5}{6} w_{1}^{2}+\frac{1}{7} w_{1}^{3}\right) .
\end{aligned}
$$

durch die Substitutionen

$$
\begin{aligned}
z_{1} & =\operatorname{Re} \sqrt{\hat{\lambda} / 8} g q_{1} \sqrt{u_{1} ;} & t_{1}=z_{1} /\left(1+\sqrt{1+z_{1}^{2}}\right) ; \\
w_{1} & =2 t_{1}{ }^{2} /\left(1-t_{1}{ }^{2}\right), &
\end{aligned}
$$

dann ergibt sich:

$$
\begin{aligned}
& J_{1}=\frac{1}{2} \frac{u_{1}^{2}}{\operatorname{Re} \sqrt{\lambda} g \sqrt{8} q_{1} \sqrt{u_{1}}} t_{1}\left(1+\frac{1}{3} t_{1}^{2}\right) ; \\
& J_{2}=\frac{1}{4} \frac{u_{1}^{4}}{\operatorname{Re} \sqrt{\lambda} g \sqrt{8} q_{1} \sqrt{u_{1}}} t_{1}\left(1+\frac{1}{5} t_{1}^{2}-\frac{1}{15} t_{1}^{4}+\frac{1}{105} t_{1}^{6}\right) .
\end{aligned}
$$

So bekommt man, wenn man $u_{2}^{2}$ und $u_{2}^{4}$ mit Hilfe von $(24 \mathrm{~b})$ umrechnet, für die Strömungskennlinie:

$$
\frac{1}{\sqrt{ } \lambda}=\frac{1}{\sqrt{ } 8 \varkappa g}\left\{\ln \left(z_{2}+\sqrt{1+z_{2}^{2}}\right)+t_{2} \cdot\left\lceil\frac{\varkappa}{4 q_{2}}\left(1-u_{1}^{4}\right)-2\right]+\left[\frac{\varkappa u_{1}^{4}}{4 q_{1} \sqrt{u_{1}}}\right] t_{1}\left(1+\frac{t_{1}^{2}}{5}-\frac{t_{1}^{4}}{15}+\frac{t_{1}^{6}}{105}\right)\right\}
$$

und für das Geschwindigkeitsverhältnis $v_{0} / \bar{v}$ :

$$
\frac{v_{0}}{\bar{v}}-1=\sqrt{\frac{\lambda}{8}} \frac{1}{x g}\left\{t_{2}\left[\frac{\varkappa}{4 q_{2}}\left(1-u_{1}^{2}\right)^{2}\right]+\left[\frac{\varkappa u_{1}^{2}}{4 q_{1} \sqrt{u_{1}}}\right] t_{1}\left(2\left(1+\frac{t_{1}^{2}}{3}\right)-u_{1}^{2}\left(1+\frac{t_{1}^{2}}{5}-\frac{t_{1}^{4}}{15}+\frac{t_{1}^{6}}{105}\right)\right)\right\} .
$$

Dabei sind die folgenden Abkürzungen benutzt:

$$
\begin{aligned}
z_{1}=\operatorname{Re} \sqrt{\lambda / 8} q_{1} \sqrt{u_{1}} g ; & z_{2}=\operatorname{Re} \sqrt{\lambda / 8} q_{2} g ; \\
t_{1}=z_{1} /\left(1+\sqrt{1+z_{1}^{2}}\right) ; & t_{2}=z_{2} /\left(1+\sqrt{1+z_{2}^{2}}\right) .
\end{aligned}
$$

Diese Formeln sehen, verglichen mit dem HagenPoiseuilleschen Gesetz (8) und der Prandtl-v. Kármánschen Formel (16), verhältnismäßig kompliziert aus. Man muß sich jedoch klarmachen, daß die in eckige Klammern gesetzten Ausdrücke Konstanten darstellen, die allerdings im folgenden noch festgelegt werden müssen, und daß beim Einsatz elektronischer Datenverarbeitungsanlagen der rechnerische Mehraufwand gegenüber den einfachen Grenzgesetzen (8) und (16) kaum merklich ins Gewicht fällt.

Da $\varkappa=0,4$ gesetzt wird und $g$ bei voller Turbulenz gleich Eins ist, bleiben in den Gln. (29) und (30) die

\begin{tabular}{lccr}
\hline$u_{1}$ & $u_{2}$ & $q_{1}$ & $q_{2}$ \\
\hline 0,773 & 0,9731 & $\infty$ & $10,779 \cdot 10^{-3}$ \\
0,78 & 0,9735 & 2,757 & $10,570 \cdot 10^{-3}$ \\
0,8 & 0,9753 & 0,7318 & $9,887 \cdot 10^{-3}$ \\
0,82 & 0,9771 & 0,4310 & $9,150 \cdot 10^{-3}$ \\
0,84 & 0,9791 & 0,3097 & $8,354 \cdot 10^{-3}$ \\
0,86 & 0,9813 & 0,2440 & $7,496 \cdot 10^{-3}$ \\
0,88 & 0,9836 & 0,2027 & $6,572 \cdot 10^{-3}$ \\
0,90 & 0,9860 & 0,1742 & $5,582 \cdot 10^{-3}$ \\
0,92 & 0,9887 & 0,1532 & $4,525 \cdot 10^{-3}$ \\
0,94 & 0,9915 & 0,1370 & $3,406 \cdot 10^{-3}$ \\
0,96 & 0,9944 & 0,1238 & $2,239 \cdot 10^{-3}$ \\
0.98 & 0,9974 & 0,1126 & $1,059 \cdot 10^{-3}$ \\
\hline
\end{tabular}

Tab. 1. Parameter zur Radialverteilung des Mischungsweges nach Anpassung an die Grenzgesetze.
Größen $u_{1}, q_{1}$ und $q_{2}$ als frei wählbare Parameter übrig. Verlangt man, daß (29) und (30) für große Reynolds-Zahlen in die Grenzgesetze (16) und (12) übergehen sollen, dann bekommt man zwei Beziehungen zwischen diesen drei Größen. Es bleibt dann nur noch ein freier Parameter, z.B. $u_{1}$, übrig. Diese Abhängigkeit der Größen $q_{1}, q_{2}$ und $u_{2}$ vom Parameter $u_{1}$ ist in Tab. 1 dargestellt.

\section{c) Erweiterung auf den gesamten Strömungsbereich. Einführung des ,Turbulenzgrades“"}

Die Lösung (29) gibt bei Verwendung der Parameter aus Tab. 1 und der Festlegung $g \equiv 1$ nur bei großen Reynolds-Zahlen die Messungen richtig wieder. Bei mittleren Reynolds-Zahlen verläuft sie wie die Kurven in den Abbildungen 5 und 8 oberhalb der Meßkurve und geht erst bei extrem kleinen Reynolds-Zahlen in den laminaren Zweig der Strömungskennlinie über. Um eine bessere Übereinstimmung zu erreichen, muß man berücksichtigen, daß der Mischungsweg auch von der Reynolds-Zahl abhängt, denn unterhalb $R e=2000 \mathrm{mu}$ dieser praktisch gleich Null sein. Dieses Ziel läßt sich durch den Produktansatz (23) für den Mischungsweg erreichen. $g$ ist dabei eine Größe, die im Übergangsgebiet von Null auf Eins ansteigt und als ,Turbulenzgrad“ bezeichnet werden soll. Bei der Herleitung eines physikalisch sinnvollen Ansatzes für diesen Turbulenz$\operatorname{grad} g$ leisten die bereits in der Einleitung erwähnte Vorstellung, daß es sich bei dem Übergang zur Tur- 
bulenz um eine Anregung höherer Energiezustände handelt, und die Analogie zur thermischen Anregung von Molekülen gute Dienste. Ist $n_{\mathrm{a}}$ die Anzahl der Atome und $n$ die Gesamtteilchenzahl pro Volumeneinheit, dann lautet das Dissoziationsgesetz:

$$
n_{\mathrm{a}}^{2} /\left(n-n_{\mathrm{a}}\right) \sim(k T)^{3 / 2} \exp \left(-E_{\mathrm{D}} / k T\right) .
$$

Dabei ist $E_{\mathrm{D}}$ die Dissoziationsenergie, und $k T$ repräsentiert die thermische Energie. Dieser thermischen Energie entspricht im vorliegenden Fall bedeutungsmäßig das Druckgefälle $\Delta p$, das dimensionsmäßig eine Energie pro Volumeneinheit ist, oder eine zu $\Delta p$ proportionale Größe. Da sich die Strömungskennlinie allein als Beziehung zwischen $\lambda$ und $R e$ darstellen läßt, liegt es nahe, den Parameter $\operatorname{Re}^{2} \cdot \lambda \sim \Delta p$ dafür heranzuziehen. Es sei also angesetzt:

$$
g /(1-g)=\left[\left(R_{e}^{2} \lambda\right)^{\alpha} / A\right] \exp \left(-E / R_{e}^{2} \lambda\right) .
$$

$E$ und $A$ sind dabei noch freie Parameter; für den Exponenten $\alpha$ seien die Werte $1 / 2$ und 1 vorgesehen. Damit ergibt sich die folgende Aufgabenstellung: Für die in Tab. 1 zusammengestellten radialen Verteilungen des Mischungsweges sollen jeweils für $\alpha=1 / 2$ und $\alpha=1$ die Parameter $E$ und $A$ so variiert werden, daß die sich ergebende analytische Lösung durch den Punkt $P_{0}$ in Abb. 11 geht und dort die richtige Tangente hat. Von allen so optimierten Kurven wird diejenige herausgesucht, die den gesamten Strömungsbereich am besten wiedergibt. Dieses Konzept läßt sich bei Einsatz einer elektro-

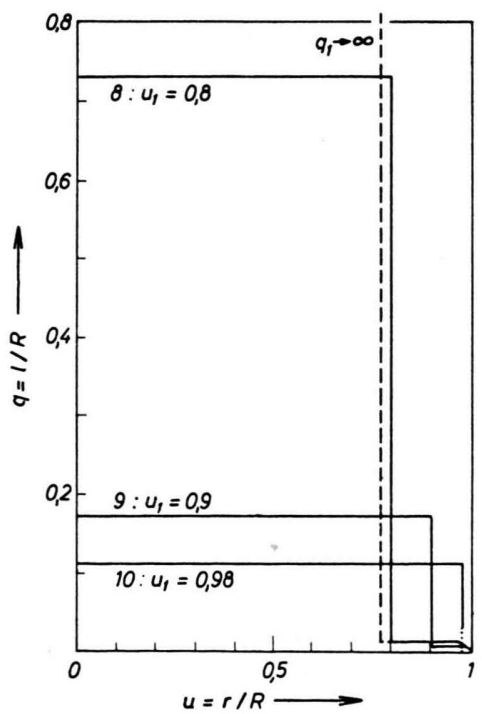

Abb. 10. Drei idealisierte Radialverteilungen des Mischungsweges zur analytischen Lösung der Gln. (21) und (22). nischen Datenverarbeitungsanlage mit durchaus endlichem Aufwand bewältigen, wenn man systematisch vorgeht. Da der Punkt $P_{0}$ durch den Parameter

$$
R e^{2} \lambda=2,56 \cdot 10^{5}
$$

gekennzeichnet ist, empfiehlt es sich, die Gl. (33) zu diesem Zweck wie folgt umzuschreiben:

$$
\begin{aligned}
& \frac{g}{1-g}=\beta\left(\frac{R e^{2} \lambda}{2,56 \cdot 10^{5}}\right)^{\alpha} \cdot \exp \left\{\gamma\left(1-\frac{2,56 \cdot 10^{5}}{R e^{2} \lambda}\right)\right\} \\
& \text { mit } \quad E=\gamma \cdot 2,56 \cdot 10^{5} \quad \text { und } \\
& A=\left(2,56 \cdot 10^{5}\right) \alpha / \beta \cdot e^{\gamma}
\end{aligned}
$$

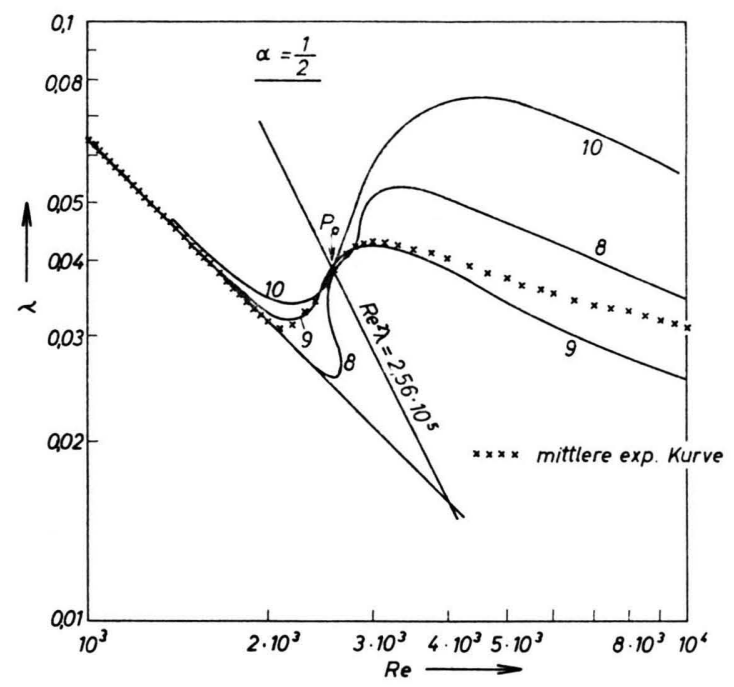

Abb. 11. Die theoretischen Strömungskennlinien zu den Verteilungen von Abb. 10 nach Optimierung des Turbulenzgrades $g$ für $\alpha=1 / 2$.

Damit erreicht man, daß $\beta$ und $\gamma$ in der Größenordnung von Eins liegen. Hält man $\beta$ fest und variiert $\gamma$, dann schneiden alle Kurven die Gerade $R e^{2} \lambda$ $=2,56 \cdot 10^{5}$ in einem Punkt, repräsentiert durch $g /(1-g)=\beta$. Man kann also zunächst für beliebiges $\gamma$ durch Variation von $\beta$ erreichen, da $\beta$ die Kurve durch den Punkt $P_{0}$ geht, und dann durch Variation von $\gamma$ für die richtige Tangente sorgen. Abb. 10 zeigt drei repräsentative Verteilungen aus Tab. 1, gekennzeichnet durch $u_{1}=0,8, \quad u_{1}=0,9$ und $u_{1}=0,98$. In Abb. 11 sind die nach dem eben geschilderten Verfahren optimierten Kurven für den Exponenten $\alpha=1 / 2$ dargestellt. Die Kurve 9 gibt zwar das Übergangsgebiet befriedigend wieder, fällt dann aber zu tief ab. Besser ist die Übereinstimmung im Fall $\alpha=1$ (Abb. 12). Hier findet man in der Kurve 9 eine Lösung, die den gesamten Strö- 


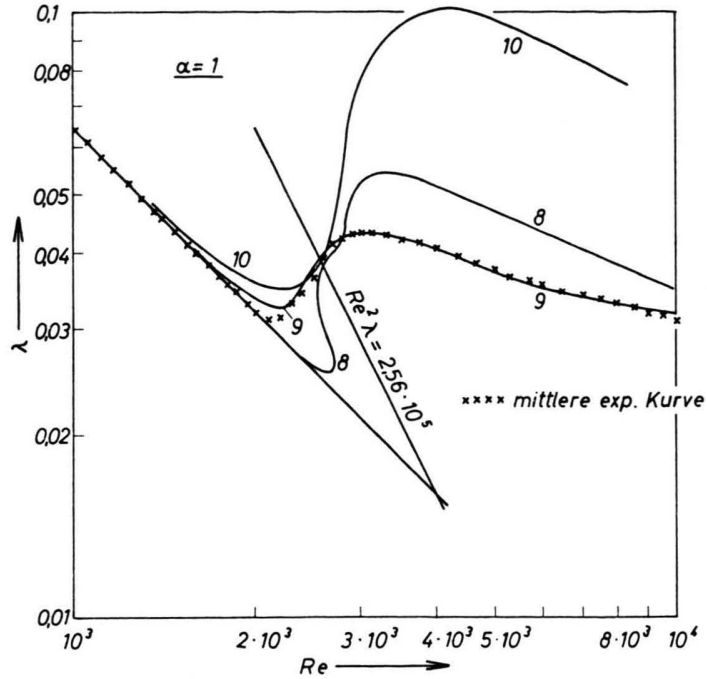

Abb. 12. Die theoretischen Strömungskennlinien zu den Verteilungen von $\mathrm{Abb} .10$ nach Optimierung des Turbulenzgrades $g$ für $\alpha=1$.

mungsbereich recht gut beschreibt. Sie ist gegeben durch die Gln. (29), (30), (31), (33) bzw. (35a, b) und die Parameterkombination:

$$
\begin{aligned}
u_{1} & =0,9 ; \quad u_{2}=0,9860 ; \quad q_{1}=0,1742 ; \\
q_{2} & =5,582 \cdot 10^{-3} ; \quad \alpha=1 ; \quad \beta=1 / 7 ; \quad(36) \\
\gamma & =0,95 ; \quad E=2,432 \cdot 10^{5} ; \quad A=6,932 \cdot 10^{5} .
\end{aligned}
$$

Setzt man diese Werte an Stelle der eckigen Klammern in (29) und (30) ein, dann bekommt die so bestimmte Lösung die Form:

$$
\begin{aligned}
& 1 / \sqrt{\lambda}=(0,8839 / g)\left\{\operatorname { l n } \left(z_{2}+\sqrt{\left.1+z_{2}^{2}\right)}\right.\right. \\
& +4,161 \cdot t_{2}+0,3969 \cdot t_{1} \\
& \left.\cdot\left(1+\frac{t_{1}^{2}}{15}-\frac{t_{1}^{4}}{15}+\frac{t_{1}^{6}}{105}\right)\right\}, \\
& v_{0} / \bar{v}-1=(0,8839 / g) \sqrt{\lambda}\left\{0,6466 \cdot t_{2}+0,4901 \cdot t_{1}\right. \\
& \left.\cdot\left(1,19+0,505 \cdot t_{1}^{2}+0,054 \cdot t_{1}^{4}-0,0077 \cdot t_{1}^{6}\right)\right\}(37 \mathrm{~b}) \\
& \text { mit } z_{1}=0,05843 \cdot \operatorname{Re} \sqrt{\lambda} g ; z_{2}=1,974 \cdot 10^{-3} \cdot \operatorname{Re} \sqrt{\lambda} g
\end{aligned}
$$

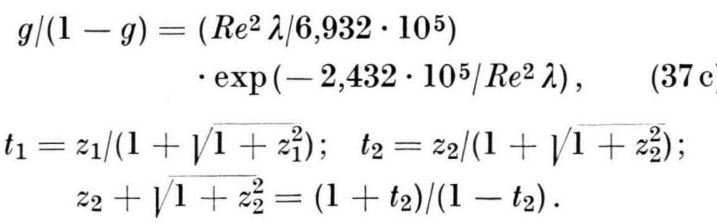

Das Hagen-Poiseuillesche Gesetz ergibt sich hieraus durch den Grenzübergang $g \rightarrow 0$, die Prandtlv. Kármánsche Formel entsprechend durch $g \rightarrow \mathbf{1}$; $\operatorname{Re} \sqrt{\lambda} \rightarrow \infty$. Da die Werte von $t_{1}$ und $t_{2}$ zwischen
0 und 1 liegen, variiert die innere Klammer in (37a) zwischen 1 und 8/7, in (37 b) zwischen 1,19 und 1,74. Diese Klammerausdrücke sind also nur wenig veränderlich und können eventuell noch etwas vereinfacht werden.

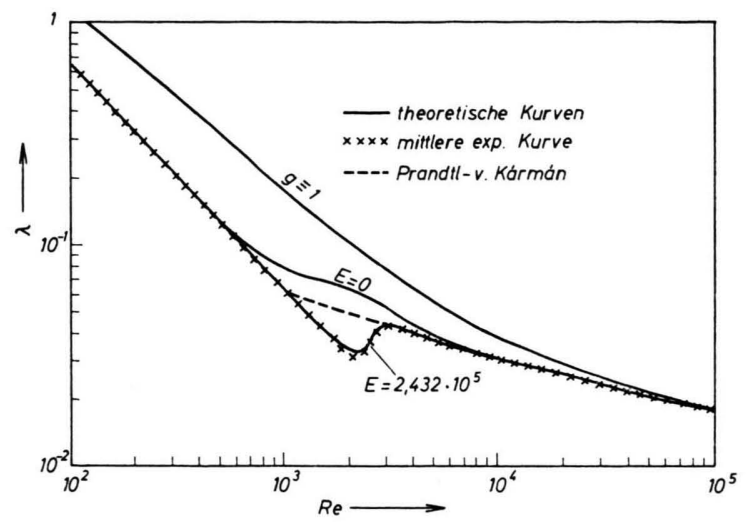

Abb. 13. Die endgültige theoretische Strömungskennlinie zusammen mit der Mittelkurve durch die Messungen von Stanton und Pannel ${ }^{7}$ und der Formel von Prandtl und v. KÁRMÁn. Eingezeichnet sind außerdem die theoretischen Strömungskennlinien, die durch $g \equiv 1$ und $E \equiv 0$ gekennzeichnet sind.

In Abb. 13 ist diese theoretische Strömungskennlinie noch einmal zusammen mit der Mittelkurve durch die Messungen von Stanton und Pannel 7 und der Prandtl-v. Kármánschen Formel abgebildet. Als Ergänzung ist die Kurve eingezeichnet, die durch den Turbulenzfaktor $g \equiv 1$ beschrieben wird, und die Kurve, die sich für $E=0$, also

$$
g /(1-g)=R e^{2} \lambda / 6,932 \cdot 10^{5}
$$

ergibt. Es sei dabei erwähnt, daß der untere Scheitelpunkt der Strömungskennlinie bei $R e=2300$ durch den Turbulenzgrad $g=0,06$, der obere Scheitelpunkt bei $R e=3000$ durch $g=0,23$ gekennzeichnet ist. Erst bei $R e=5 \cdot 10^{4}$ ist der Turbulenzgrad $g=1$ etwa erreicht. Damit wird der bereits in Abschnitt I erwähnte Effekt deutlich. Setzt man in der genauen Lösung den Turbulenzgrad $g$ gleich Eins, dann bekommt man eine wesentlich schlechter passende Kurve. Geht man in der so vereinfachten Formel außerdem noch durch $R e \sqrt{\lambda} \rightarrow \infty$ zur Asymptote über, dann macht man einen zweiten Fehler, der den ersten oberhalb $R e=3000$ weitgehend kompensiert. Durch diesen Umstand bekommt die Prandtlv. Kármánsche Formel einen Genauigkeitswert, der ihr auf Grund der in ihr steckenden einfachen Annahmen eigentlich nicht zukommt. 
In Abb. 14 ist das Verhältnis von Maximalgeschwindigkeit $v_{0}$ zur mittleren Geschwindigkeit $\bar{v}$ als Funktion der Reynolds-Zahl für die drei Fälle aus Abb. 13 wiedergegeben. Eine Ergänzung zu diesen

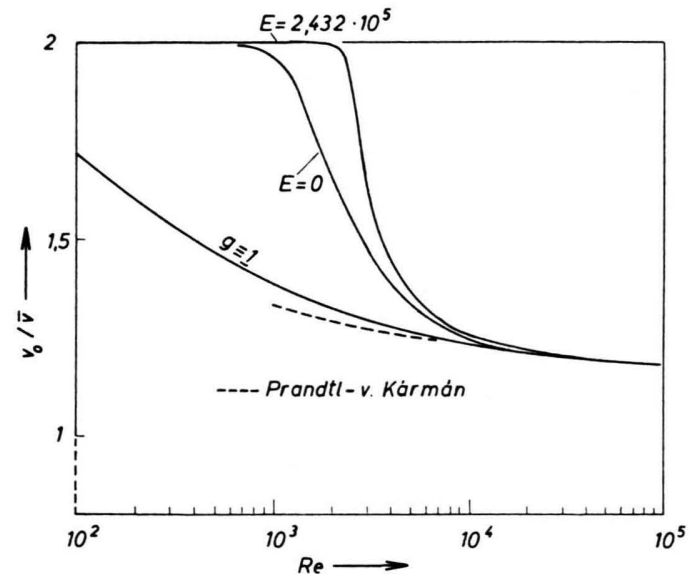

Abb. 14. Die zu den theoretischen Strömungskennlinien von Abb. 13 gehörenden Geschwindigkeitsverhältnisse $v_{0} / \bar{v}$.

Überlegungen enthält die Abb. 15. Bei der Diskussion der Zusatzeffekte an Rohrverengungen, Rohrerweiterungen, Verzweigungen usw. ${ }^{1,2}$ spielen der Impulsstrom $\int \varrho v v \mathrm{~d} f$ und der Energiestrom

$$
\int \frac{1}{2} \varrho v^{2} v \mathrm{~d} f
$$

$-\mathrm{d} f$ bezeichnet das Integral über den Querschnitt - eine große Rolle. Da z.B. der Impulsstrom nicht

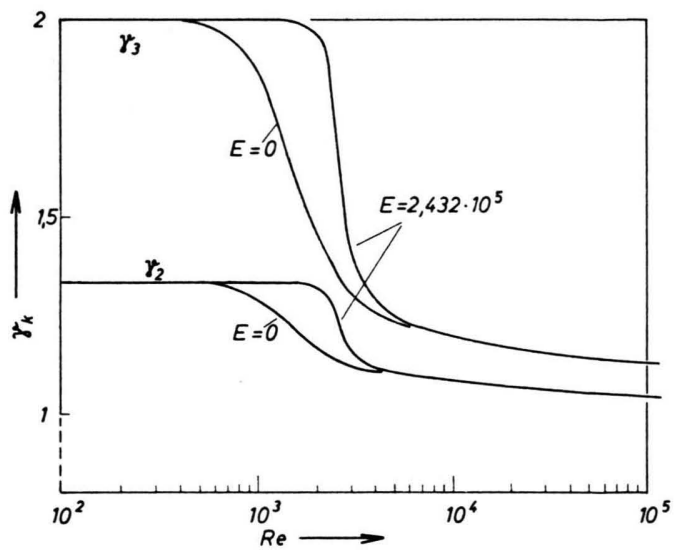

Abb. 15. Die Korrekturfaktoren $\gamma_{k}$ nach Gl. (39) für den Impuls- und den Energiestrom.

einfach gleich $\varrho \bar{v}^{2} F$ gesetzt werden kann, ist die $\mathrm{Ab}$ hängigkeit der Korrekturfaktoren

$$
\gamma_{\mathrm{k}}=\frac{\overline{v^{k}}}{\bar{v}^{k}}=\frac{\int_{0}^{R^{2}} d\left(r^{2}\right) v^{k}}{R^{2} \bar{v}^{k}}=\int_{0}^{1} \mathrm{~d}\left(u^{2}\right)[w(u)]^{k}
$$

von der Reynolds-Zahl von Interesse. Diese Beziehung ist für $\gamma_{2}$ und $\gamma_{3}$ in der Abb. 15 dargestellt.

Bei verhältnismäßig kurzen Rohren machen die Ein- und Ausströmeffekte einen wesentlichen Anteil des Druckgefälles aus. Die Rohre werden weitgehend turbulent durchströmt. Dabei hat die Druckerhöhung denselben Einfluß auf das Verhältnis $E / \Delta p$ in Gl. (33) wie eine Erniedrigung der Anregungsenergie $E$. Deshalb ist die durch $E=0$ gekennzeichnete Lösung die geeignete Grundlage zur Beschreibung dieser Zusatzeffekte. Sie kann als Lösung für die ,,gestörte Rohrströmung" bezeichnet werden ${ }^{24}$.

\section{Zusammenfassung}

Die Aufgabe, eine analytische Lösung für die Strömungskennlinie und das Verhältnis von Maximalgeschwindigkeit $v_{0}$ zur mittleren Geschwindigkeit $\bar{v}$ herzuleiten, besteht in der Hauptsache in der Festlegung des Mischungsweges $l$, der den turbulenten Beitrag zur Schubspannung bestimmt. In der vorliegenden Arbeit wird die Abhängigkeit des Mischungsweges von der radialen Ortskoordinate und der Reynolds-Zahl durch einen Produktansatz beschrieben. Die Forderung, daß die theoretische Strömungskennlinie und das Geschwindigkeitsverhältnis $v_{0} / \bar{v}$ in die von Prandtl und v. Kármán hergeleiteten und experimentell bestätigten Grenzgesetze übergehen müssen, legt den radialen Anteil des Produktansatzes fest. Der von der ReynoldsZahl abhängige Faktor dieses Produktes steigt im Übergangsgebiet zwischen laminarer Strömung und voller Turbulenz von Null auf Eins an und wird hier ,,Turbulenzgrad“ genannt. Die Analogie zum Dissoziationsgrad als einem Ausdruck für die thermische Anregung höherer Energiezustände führt auf einen physikalisch sinnvollen Ansatz für diesen Faktor. Die darin noch freien Parameter werden durch optimale Anpassung der theoretischen Kurve im Übergangsgebiet an die von Stanton und PanNeL ${ }^{7}$ gemessene Strömungskennlinie festgelegt. Obwohl die hier benutzten phänomenologischen Ansätze in $u_{1}, u_{2}, q_{1}, E, A$ und $\alpha$ sechs freie Parameter enthalten, führt das beschriebene Verfahren zur optimalen Anpassung an die Messungen zu einer eindeutigen Bestimmung aller Größen. Der hier beschriebene Ansatz scheint daher also der einfachst mögliche zu sein, der es erlaubt, die Strömungskennlinie in solch guter Übereinstimmung von Theorie und Messung darzustellen.

24 W. Frie u. Рh. Jäger, Z. Naturforsch., im Druck. 